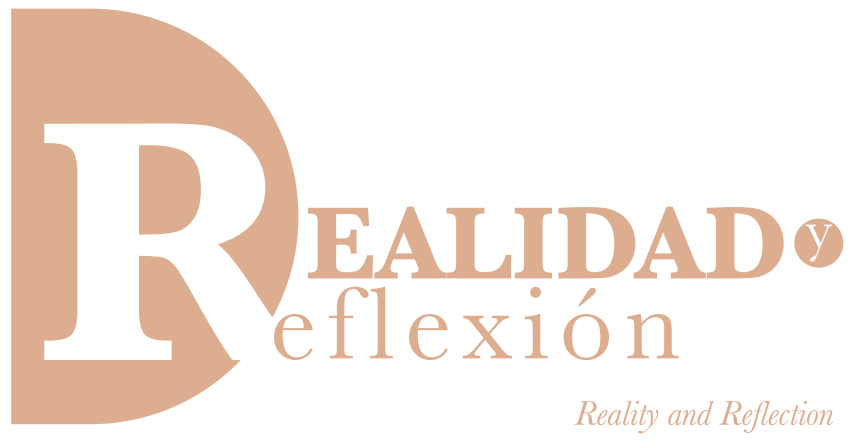

ISSN 1992-6510

e-ISSN 2520-9299

Año 17, N 46, San Salvador, El Salvador, Centroamérica. Revista Semestral Julio-Diciembre 2017

YEAR 17, N 46, SAN SALVADor, El SALVADoR, CENTRAL AMERICA. SEMESTRAL JoURNAL JULY-DECEMBER 2017

\title{
100 circuitos escolares vivificantes de la reforma educativa de 1968
}

\author{
100 vivifying school circuits of the 1968 \\ educational reform
}

\author{
Carmen Etelvina Rubio Sorto de Peña ${ }^{1}$ \\ Licenciada en Ciencias de la Educación, Master en Docencia y Administración Universitaria \\ por la Universidad "Doctor Manuel Luis Escamilla" de El Salvador. \\ Docente del Complejo Educativo "Concha Viuda de Escalón" \\ cers100@hotmail.com \\ Recibido: 18 de septiembre de 2017 \\ Aprobado: 7 de noviembre de 2017 \\ pág. $76-82$ \\ DOI: http://dx.doi.org/10.5377/ryr.v0i46.5509
}

\section{RESUMEN}

Ensayo sobre la Reforma Educativa de 1968, la creación de 100 Circuitos Escolares y la función administrativa y pedagógica de 100 Supervisores docentes preparados por el sistema en la Ciudad Normal "Alberto Masferrer" de la República de El Salvador, para el cumplimiento de la misión y visión de la Reforma Educativa de 1968, durante la gestión ministerial del licenciado Walter Béneke Medina y el Viceministro de la misma cartera de Estado, licenciado Roberto Murray Meza, y el licenciado Carlos De Sola, encargado de la Dirección Nacional de Cultura y Juventud.

Palabras clave: Reforma educativa, 1968, supervisores, escolares.

\section{ABSTRACT}

Essay on the Educational Reform of 1968, the Creation of 100 School Circuits and the Administrative and Pedagogical Function of 100 School Supervisors prepared by The System in the Normal City "Alberto Masterrer" of the Republic of El Salvador, for the fulfillment of the mission and vision of the Educational Reform of 1968, during the Ministerial Management of Mr. Walter Béneke Medina and the Deputy Minister of the same State Portfolio, Mr. Roberto Murray Meza, and Mr. Carlos de Sola, in charge of the National Directorate of Culture and Youth.

Keywords: Educational reform, 1968, school, supervisors.

1 (C) Carmen Etelvina Rubio Sorto de Peña 
Partiendo del axioma de que el método de enseñanza-aprendizaje reforma el sistema educativo. A mediados de 1968 se convocó a 150 maestros y maestras a presentarse a la Ciudad Normal "Alberto Masferrer", con la finalidad de recibir una capacitación sobre lo que debería ser la nueva forma de educar en las escuelas públicas, con aspectos novedosos en la metodología de la planificación y desarrollo programático de las cuatro asignaturas de la educación básica; y que debería ser el corazón de la nueva escuela salvadoreña, conmocionando su organización administrativa y de servicio pedagógico de todo el sistema educativo.

Estos 150 educadores y educadoras convocados y provenientes de distintas zonas del país que fueron seleccionados según el criterio de los entonces Delegados Escolares, se convertirían en el selecto alumnado para conocer y remozar los apoyos metodológicos en la nueva enseñanza sobre: teoría de conjuntos en la matemática moderna; la gramática estructural, en el caso de la asignatura de Idioma Nacional; en la práctica de viveros y huertos escolares en las Ciencias Naturales; la importante participación de alumnos y alumnas en dramatizaciones y el encuentro de líderes en Estudios Sociales y desde luego, la práctica sistemática de la Educación Física; la Educación Musical, a través de estudiantinas, coros, etc., como parte de esta inicial capacitación hacia la "Reforma educativa" de 1968.

Cada uno de los asistentes recibió en primer lugar, la cordial bienvenida del entonces señor director de la Ciudad Normal "Alberto Masferrer", Maestro y licenciado en Sociología, don Gilberto Aguilar Avilés, y del Sr. Subdirector de la misma, también licenciado, Ricardo Mena López. En segundo lugar, la formación de parte de un equipo de catedráticos especializados en enseñar cómo lograr los objetivos educacionales en el alumnado. En tercer lugar, el importante contenido didáctico, pedagógico, metodológico, integrado o concentrado en los textos, de 60 libros o documentos donados por la UNICEF, entre los cuales estaban: La querella de los métodos, Cómo enseñar a leer con el método global, Cómo mejorar los programas escolares, Experimentos básicos en las Ciencias Naturales, Gramática estructural, la Psicología Social, entre otros.

Terminado ese periodo, a manera de un momento propedéutico, fueron despedidos para llevar consigo los conocimientos y la noticia a sus respectivos centros escolares, sobre la puesta en común de lo que en un momento determinado traería la nueva forma de enseñar, con un nuevo recurso metodológico y dinamizador de cada clase, transformando operativamente el ambiente del aula y fuera de ella, dándole un fuerte valor a la importancia del desarrollo físico, psicológico e intelectual del alumnado. Estos 150 maestros y maestras llevaron el mensaje de lo que tendría que ser la Reforma Educativa de 1968.

Ese mismo año, se hizo un nuevo llamado para un número de 50 educadores y educadoras, de los 150 que tuvieron la experiencia propedéutica, para un periodo de otros tres meses en la Ciudad Normal "Alberto Masferrer".

En la inauguración de ese nuevo e importante encuentro se tuvo la sorpresa de contar con el Ministro de Educación, el licenciado Walter Béneke Medina. 
En su exposición de bienvenida y justificación del curso, destacó que el objetivo era preparar y seleccionar a los que deberían ser los 20 nuevos Supervisores Docentes que la Reforma Educativa necesitaba, para complementar los 100 profesionales de la educación que debían asumir la responsabilidad de conducir la administración y servicio técnico a igual número de Circuitos Escolares, ya calculados y planificados para el servicio educativo a nivel nacional.

Hizo la invitación a que en esos tres meses todos brindaran especial dedicación para que los 20 nuevos supervisores tuvieran el mejor rendimiento educacional, de acuerdo a los criterios del curso y a una conducta moral satisfactoria. Ellos cubrirían las plazas vacantes.

En marzo de 1969 se cumplió el nombramiento de los nuevos 20 Supervisores Docentes.

E1 profesor Carlos Gutiérrez, Supervisor General de Educación, en una sala del edificio de la Biblioteca Nacional, que era ocupado por la mayoría de las dependencias del Ministerio de Educación, presentó los respectivos nombramientos a los 20 profesores convocados.

El señor Supervisor General los felicitó por el grado escalafonario alcanzado y les deseó éxitos en el cumplimiento educativo que el sistema requería.

Explica el profesor Gutiérrez, que en la zona occidental, en especial en el departamento de Santa Ana, se asignaba a los supervisores con la mejor valoración de desempeño, obtenido durante las dos jornadas realizadas. En ese cuadro de nuevos nombramientos se encontraban:
Raúl Antonio Peña, Roberto Guevara, Miguel Ramírez Gómez, Berta Alicia Linares de Rodríguez, Remberto Arana Barrientos.

Para otros lugares como Sonsonate, La Libertad, Morazán, San Salvador, La Unión y Chalatenango, se nombraron a: José Manuel Calzadilla, Daniel Humberto Marenco, Rubén Orellana Mendoza, Pablo Soriano, Roberto Rodolfo Rodríguez, Ulises Portillo, Héctor Antonio Pérez, Luis Antonio Amaya, Ricardo Jiménez, Reynaldo Reyes, Ennio Guinea, José del Carmen Parada, René Argueta Barillas, Francisca de Romero y Manuel Quijada.

Para el año de 1970, siempre en la Ciudad Normal "Alberto Masferrer", se convocó a todo el cuerpo de Supervisores Docentes, 100 en total, para una formación competitiva hacia la docencia de nivel superior que duraría nueve meses.

En la Ciudad Normal, estos profesionales gozaron de alimentación, alojamiento, uso de la Biblioteca, de los amplios salones de clase, dormitorios, un excelente auditórium, zonas de recreo, campo deportivo, así como también de transporte desde Ciudad Normal hacia San Salvador; disponible para todos los alumnos que desearan hacer uso de ellos y otros anexos. Las jornadas de estudio diarias se iniciaban a las 8:00 a. m. y concluían al medio día, para luego ser reanudadas de 1:30 p. m. a las 4:00 p.m.

Entre los Supervisores Docentes de esta nueva e importante etapa para la Reforma Educativa de 1968, estuvieron: Adalberto Quinteros Chulo, Oscar Francisco Agreda, Héctor Eduardo Cañas, Hernán Villegas Amaya, Noé Isaac Espinoza, Roberto Campos Tenorio, 
Antonio Ulloa, Jaime Israel Pimentel, Francisco Escobar, Oscar Martínez, Romeo Peña Gómez, Evaristo Molina, César Augusto Bolaños, Eufrasio Chévez, Salvador Chévez, Amadeo Armando Machuca, Luis Alonzo Reyes, Julio César Martínez, Candelario Vargas, Erlinda Hernández de Mora, Rigoberto Aguilar Guido, Tito Vega, Mardoqueo Hernández, David Colato, Guillermo Fuentes, José Álvaro Pacheco, José Ovidio Ramos, entre otros; y además de los nuevos 20 supervisores docentes nombrados en el mes de marzo de 1969.

Los 100 Supervisores Docentes tenían como jefe inmediato un Supervisor Itinerante para cada 10 de ellos, recordando a don Armando Martí y Julio Castillo Orantes.

Así fue como, en ese año de 1969, la Reforma Educativa dio a luz administrativamente a los 100 Circuitos Escolares, los 100 Supervisores Docentes, que a nivel nacional hicieran los debidos cumplimientos en la dimensión cualitativa que el Sistema de Educación requería.

El compromiso de estos 100 profesionales se centró en lo siguiente:

1. Nuevos programas curriculares, con un cuerpo textual de precisos y claros objetivos a lograr, contenidos y sugerencias metodológicas; juicios valorativos para la correcta aplicación de la actividad a evaluar, tiempos y recursos de apoyos didácticos, todo ello reclamado y programado en una planificación hecha por el profesional docente, con criterio pedagógico y con apego a la realidad circundante para ser aprovechada o para superar la incidencia de ella en los fines, objetivos y metas, visionados como logros a futuro. Este aspecto de la nueva escuela salvadoreña con relación a los programas escolares puso en acción la vocación y la capacidad más profesional del magisterio.

2. La modulación de los programas de grado, permitían su aplicación temática de un grado inferior hacia el subsiguiente grado, a tal punto que el conocer el desarrollo de cada alumno por parte del docente, favorecía el hecho de cómo llevar la clase al siguiente grado, porque ese mismo docente tenía la responsabilidad del grado en el nuevo año lectivo.

3. El conocimiento personalizado de la clase haría alcanzables los objetivos filosóficos de la llamada "Promoción Orientada", para no tener alumnos reprobados, dado que ello afectaría psicológicamente y socialmente tanto al estudiante como a su núcleo familiar; y a su vez, económicamente al hogar y al Estado salvadoreño; con el agravante de los señalamientos negativos sobre la calidad del centro escolar y sus docentes.

4. Las dos escuelas experimentales de E1 Salvador y sus actividades, aplicadas en las llamadas Escuelas Renovadas, se convirtieron en ejemplos de buenas prácticas y a su vez, en promotoras de los beneficios obtenidos, en provecho de todas las escuelas salvadoreñas.

5. La Reforma Educativa de 1968 fue conjugada en parte con la experiencia 
de los directores de estos centros experimentales: la Escuela Experimental Dr. Humberto Romero Alvergue, en San Salvadory la Escuela ExperimentalTomás Medina, en Santa Ana, precisamente en la colonia El Palmar.

6. Fueron fundamentales los aportes de la experiencia del licenciado Augusto Aguilar Ferrufino, director de la Escuela Experimental "Doctor Humberto Romero Alvergue" y del profesor Ismael Armando Villa, director de la Escuela Experimental “Tomas Medina”.

7. De las escuelas renovadas se recibió el apoyo de algunos miembros de su planta docente, algunos de los cuales clasificaron como Supervisores Docentes. Entre las Escuelas Renovadas se citan las siguientes: Escuela Renovada "José Pantoja”, en La Unión; Escuela Renovada "Darío González", de San Vicente; Escuela Renovada "Ofelia Herrera", de San Miguel; Escuela Renovada "Anita Alvarado" de Cojutepeque; Escuela Renovada "Napoleón Ríos", de Santa Ana y Escuela Renovada de Varones de Chalatenango, más el aporte de otras de igual nivel y calidad educativa.

8. El cometido de estos centros escolares, en su experiencia de trabajo con "Centros de Interés", "Unidades de Trabajo", "Estudio de Casos", dio justificación a su presencia en el marco de la Reforma Educativa de 1968, que buscaba una nueva forma de planificar y desarrollar la atención a los escolares.
9. El hecho de dinamizar el entorno de la enseñanza-aprendizaje ya fuese dentro o fuera de la escuela, tenía que estar enmarcado en una motivación constante y facilitadora del aprendizaje.

10. Los Supervisores Docentes, en su totalidad y con sus capacidades profesionales del quehacer administrativo y pedagógico, fueron conocedores y partícipes de la encomendada misión y visión estructural de la Reforma Educativa de ese año, cada quien en su inmediata responsabilidad de su Circuito Escolar.

\section{Funciones del Supervisor Docente:}

A. Conocer la planta docente de cada una de sus escuelas, con detalles importantísimos como la clásica teoría de los educadores; la razón del grado o clase asignada; los tiempos de sus ascensos escalafonarios e inclusive otros datos personales de interés.

B. Integrar sus acciones como Supervisores para impulsar el cambio necesario en la escuela salvadoreña, en sus estructuras administrativa y pedagógica, cumpliendo, desde luego, los ideales o fines de la Reforma Educativa. Relacionarse efectivamente con la comunidad sin perder de vista la importante conexión social entre las autoridades locales, los padres y madres de familia, los clubes de servicio, conociendo además el patrimonio de la comunidad, su identidad cultural y otros aspectos que favorecieran la misma existencia novedosa de la Reforma Educativa. 
C. Apoyar y resolver en beneficio de la creación de las Casas de la Cultura, los Círculos Estudiantiles, la unificación de la educación básica, la creación de la diversificación del nivel superior escolar, la fundación de las Escuelas tipo 3-3-6 en el área rural del país; fortalecer el incremento matricular y mantener dicha matrícula hasta el nivel básico de nueve grados; y desde luego, justificar la no existencia de los exámenes privados para los alumnos por egresar de bachillerato, dado que la historia en este punto recogía más que éxitos, tristeza en los hogares.

La Reforma Educativa de 1968 caminó con la exactitud del desarrollo de planes y programas, mediante el soporte profesional y la experiencia de 100 Supervisores Docentes que aún ante las circunstancias de origen político y otras de conquista magisterial, alcanzaron grandes logros que hacia los años del nuevo siglo se mantienen como símbolo de la inequívoca misión cultural de aquellos tiempos. Es innegable el hecho de que el origen de adelantos educativos es en parte creado por ese cambio metodológico en la educación y que así mismo, cambió la estructura administrativa del Sistema Educativo para el mejoramiento del parámetro como indicador del desarrollo de la escolaridad básica, superior y universitaria entre otras.

Es de obligada presencia el contenido de aquella reforma con relación a la creación de la Televisión Educativa cuyo fin fue siempre servir de apoyo didáctico referencial a los docentes, en contenidos $\tan$ importantes sobre Estudios Sociales, Matemáticas, y las demás materias básicas. Este medio de comunicación permitió llevar a cabo muchos concursos escolares de dramatización, música y otros elementos culturales.

\section{Novedades como producto de la Reforma Educativa de 1968}

La escuela tradicional, con la Reforma Educativa de 1968, queda superada mediante la escuela activa y reformadora del aula y sus funciones, como resultado de una metodología de enseñanza-aprendizaje dinámica, consecuente $y$ lógica ante la persona.

La escuela tradicional tuvo que sacudir sus entrañas para un nuevo ser educativo que trajo su forma especial de presentarse a través de:

- Verdaderos currículos escolares.

- Profesionales de la educación bien calificados en su área de servicio administrativo y pedagógico.

- Apoyos circundantes de carácter escolar como: Casas de la Cultura y los Círculos Estudiantiles.

- Programas específicos de Educación Física, con maestros formados para ello.

- Programación puntual sobre expresiones culturales como: Concursos nacionales de Oratoria, de Escrituray Ortografía, concursos de Coros Estudiantiles, concursos de Estudiantinas y otros de carácter deportivo, y todos a nivel de Departamento, Zonas y Nacional.

- Las escuelas 3-3-6 eran atendidas por tres educadores, en tres salones de clases y los seis grados en turnos matutinos y vespertinos. Como la estrategia de este tipo de escuela fue de extender los servicios de la educación a zonas rurales, se planificó y ejecutó que los edificios de dichas escuelas contaran con una cocina y otros 
dos salones más que sirvieran como dormitorio para los profesores. Además contaron siempre con servicios básicos sanitarios.

- Los maestros o maestras de estas escuelas 3-36 gozaron de un sobresueldo por la atención de dos secciones, así como el correspondiente sobresueldo por ruralidad.

- Se fundaron los Bachilleratos Diversificados con tres años de duración en las áreas de Navegación y Pesca, Turismo, Salud y Mecánica Automotriz.

Como un resumen a todo lo expuesto en el presente ensayo, se confirma que el método globalizante para la enseñanza del aprendizaje obligó todas las modificaciones administrativas del Ministerio de Educación, así como la conducta de sus funcionarios, para desarrollar la teoría filosófica de la Reforma Educativa, tan necesaria para el bien de toda la escolaridad salvadoreña y así asegurar un mejor futuro para El Salvador.

Es lógico pensar que la superestructura de este Ministerio o el recurso humano del mismo, estuvo a la altura de la misión y de la visión de dicha reforma.

Puede citarse que el señor Ministro de Educación, Walter Béneke Medina, tuvo el apoyo del viceministro Roberto Murray Meza; del encargado de Arte y Cultura de esa época, el licenciado Carlos De Sola, del señor director de Educación, profesor Manuel Vela, del supervisor General de Educación, profesor Carlos Gutiérrez, del Coordinador de dicho departamento, profesor Mario Orellana y de todas las jefaturas y dependencias claves en el desarrollo de esta magna actividad nacional.

Los Supervisores Docentes de los años 69 y 70 fueron hombres y mujeres claves al servicio del desarrollo pedagógico nacional, con mucha atención a la aplicación de los currículos escolares.

Se cierra el presente ensayo, con la fotografía de algunos de los Supervisores del año de 1970, cuando concluyeron su capacitación de nueve meses en la Ciudad Normal "Alberto Masferrer". Ellos además de lo aprendido, llevaron la impresión satisfactoria de haber tenido como instructores a los licenciados Oscar Ramírez Pérez, Salvador Fonseca, los doctores Renee B. Piris y Bruno Stiglitz, entre otros.

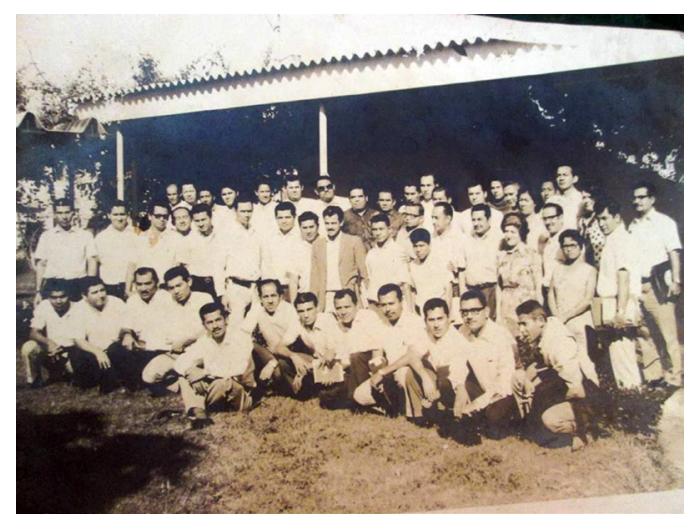

Imagen n. ${ }^{\circ}$ 1. Supervisores de 1970. 